\title{
A New Form of the General Solution of the Elastic Space Axisymmetric Problem in Pavement Mechanics
}

\author{
Jia Liang, Guo Jian, and He Shikai \\ College of Civil Engineering, Lanzhou University of Technology, Lanzhou 730050, China \\ Correspondence should be addressed to Jia Liang; jialiang1949@163.com
}

Received 20 April 2016; Revised 30 June 2016; Accepted 30 June 2016

Academic Editor: Chaudry M. Khalique

Copyright (C) 2016 Jia Liang et al. This is an open access article distributed under the Creative Commons Attribution License, which permits unrestricted use, distribution, and reproduction in any medium, provided the original work is properly cited.

In order to analyze the stress and displacement of pavement, a new form of the general solution of the elastic space axisymmetric problem is proposed by the method of mathematics reasoning. Depending on the displacement function put forward by Southwell, displacement function is derived based on Hankel transform and inverse Hankel transform. A new form of the general solution of the elastic space axisymmetric problem has been set up according to a few basic equations as the geometric equations, constitutive equations, and equilibrium equations. The present solution applies to elastic half-space foundation and Winkler foundation; the stress and displacement of pavement are obtained by mathematical deduction. The example results show that the proposed method is practically feasible.

\section{Introduction}

Winkler foundation and elastic half-space foundation have been widely used since Boussinseq represented the solution of the elastic space axisymmetric problem $[1,2]$. Study of solutions for elastic space axisymmetric problem is of great interest for a number of researchers. Westergard gave the stress analysis of concrete pavement [3]. Love has obtained the approximate solution in the elastic half-space [4]. Cerruti presented solution of stress and displacement of elastic halfspace [5]. Bagisbaev [6] and Rizzo and Shippy [7] made a study of the fundamental solution of axisymmetric elasticity problems. The common solutions of axisymmetric elastic space are Love solution and Southwell solution [8-11]. The idea of generalized images is applied to solve a contact problem [12]. Solution of a thin layer bonded on a viscoelastic medium is presented [13]. Green's functions are obtained for an infinite prestressed thin plate on an elastic foundation under axisymmetric loading [14].

Great research achievements have been obtained for axisymmetric half-space contact problems. In this paper the potential method will be developed from the stage to which it has been carried previously, and a new form of the general solution has been set up. The relationship between the traditional and the present method is discussed in the last section.

This paper is organized as follows. In Section 2, a brief description is given of the fundamental equations and the new form of general solution. Section 3 is aimed at deriving the formulas for the relationship between general solutions. Sections 4 and 5 apply the new form of the general solution to elastic half-space foundation and Winkler foundation, and in Section 6 we finish with some concluding remarks.

\section{General Solutions}

For the elastic space axisymmetric contact problems, in the cylindrical coordinates (with the $z$-axis being positioned normal to the plane of isotropy), the fundamental equations can be rewritten in the following manner.

Equilibrium equations are as follows:

$$
\begin{aligned}
\frac{\partial \sigma_{r}}{\partial r}+\frac{\partial \tau_{z r}}{\partial z}+\frac{\sigma_{r}-\sigma_{\theta}}{r} & =0, \\
\frac{\partial \sigma_{z}}{\partial z}+\frac{\partial \tau_{z r}}{\partial r}+\frac{\tau_{z r}}{r} & =0 .
\end{aligned}
$$


Geometric equations are as follows:

$$
\begin{aligned}
\varepsilon_{r} & =\frac{\partial u}{\partial r}, \\
\varepsilon_{\theta} & =\frac{u}{r}, \\
\varepsilon_{z} & =\frac{\partial w}{\partial z}, \\
\gamma_{z r} & =\frac{\partial w}{\partial r}+\frac{\partial u}{\partial z} .
\end{aligned}
$$

Constitutive equations are as follows:

$$
\begin{aligned}
\varepsilon_{r} & =\frac{1}{E}\left[\sigma_{r}-\mu\left(\sigma_{\theta}+\sigma_{z}\right)\right], \\
\varepsilon_{\theta} & =\frac{1}{E}\left[\sigma_{\theta}-\mu\left(\sigma_{r}+\sigma_{z}\right)\right], \\
\varepsilon_{z} & =\frac{1}{E}\left[\sigma_{z}-\mu\left(\sigma_{r}+\sigma_{\theta}\right)\right], \\
\gamma_{z r} & =\frac{1}{G} \tau_{z r},
\end{aligned}
$$

where $E$ is elasticity modulus; $\mu$ is Poisson's ratio; $u$ and $w$ are displacement; $G$ is shear modulus.

Compatibility equation is as follows:

$$
\begin{aligned}
\nabla^{2} \sigma_{r}-\frac{2}{r^{2}}\left(\sigma_{r}-\sigma_{\theta}\right)+\frac{1}{1+\mu} \frac{\partial^{2} \Theta}{\partial r^{2}} & =0 \\
\nabla^{2} \sigma_{\theta}-\frac{2}{r^{2}}\left(\sigma_{r}-\sigma_{\theta}\right)+\frac{1}{1+\mu} \frac{1}{r} \frac{\partial \Theta}{\partial r} & =0 \\
\nabla^{2} \sigma_{z}+\frac{1}{1+\mu} \frac{\partial^{2} \Theta}{\partial r^{2}} & =0 \\
\nabla^{2} \tau_{z r}-\frac{\tau_{z r}}{r^{2}}+\frac{1}{1+\mu} \frac{\partial^{2} \Theta}{\partial r \partial z} & =0
\end{aligned}
$$

where $\nabla^{2}=\partial^{2} / \partial r^{2}+(1 / r)(\partial / \partial r)+\partial^{2} / \partial z^{2}, \Theta=\sigma_{r}+\sigma_{\theta}+\sigma_{z}$.

We introduced $\chi(r, z)$ of the Southwell displacement function [1]; the stress can be obtained as follows:

$$
\begin{aligned}
& \sigma_{r}=\frac{\partial}{\partial z}\left(\mu \nabla^{2} \chi-\frac{\partial^{2} \chi}{\partial r^{2}}\right) \\
& \sigma_{\theta}=\frac{\partial}{\partial z}\left(\mu \nabla^{2} \chi-\frac{1}{r} \frac{\partial \chi}{\partial r}\right), \\
& \sigma_{z}=\frac{\partial}{\partial z}\left[(2-\mu) \nabla^{2} \chi-\frac{\partial^{2} \chi}{\partial z^{2}}\right], \\
& \tau_{z r}=\tau_{r z}=\frac{\partial}{\partial r}\left[(1-\mu) \nabla^{2} \chi-\frac{\partial^{2} \chi}{\partial z^{2}}\right]
\end{aligned}
$$

Substituting (5) into (1) and (4) yields

$$
\nabla^{2} \nabla^{2} \chi=0
$$

According to (2), (3), and (5), the displacement components can be expressed as

$$
\begin{aligned}
& u=\frac{1+\mu}{E} \frac{1}{r}\left(\frac{\partial^{2} \chi}{\partial z^{2}}-2 \mu v^{2} \chi\right), \\
& w=\frac{1+\mu}{E} \frac{1}{r} \frac{\partial}{\partial z}\left(\frac{\partial^{2} \chi}{\partial z^{2}}-2 v^{2} \chi\right),
\end{aligned}
$$

where $v^{2}=\partial / \partial r^{2}-(1 / r)(\partial / \partial r)+\partial / \partial z^{2}$ and $v^{2}$ is the Southwell operator.

From (2), (3), and (7), the expression of displacement component and stress component can be obtained as follows:

$$
\begin{aligned}
& \sigma_{r}=\frac{1}{r} \frac{\partial}{\partial r}\left(\frac{\partial^{2} \chi}{\partial z^{2}}-\mu v^{2} \chi\right)-\frac{1}{r^{2}}\left(\frac{\partial^{2} \chi}{\partial z^{2}}-2 \mu v^{2} \chi\right), \\
& \sigma_{\theta}=\frac{\mu}{r} \frac{\partial v^{2} \chi}{\partial r}+\frac{1}{r^{2}}\left(\frac{\partial^{2} \chi}{\partial z^{2}}-2 \mu v^{2} \chi\right), \\
& \sigma_{z}=-\frac{1}{r} \frac{\partial}{\partial r}\left[\frac{\partial^{2} \chi}{\partial z^{2}}-(1+\mu) v^{2} \chi\right], \\
& \tau_{z r}=\frac{1}{r} \frac{\partial}{\partial z}\left[\frac{\partial^{2} \chi}{\partial z^{2}}-(1+\mu) v^{2} \chi\right] .
\end{aligned}
$$

Substituting (8)-(11) into (1), displacement must satisfy the following equation:

$$
v^{4} \chi=0
$$

We can obtain (13) based on Hankel transform:

$$
\chi(r, z)=\int_{0}^{\infty} \bar{\chi}(\xi, z) J_{1}(\xi r) r d \xi
$$

Equation (13) can be rewritten as follows based on inverse Hankel transform:

$$
\bar{\chi}(\xi, z)=\int_{0}^{\infty} \chi(r, z) J_{1}(\xi r) \xi d r .
$$

A differentiation action is performed on (13), on both sides of $r$ and $z$, and we can get the following equations:

$$
\begin{aligned}
\frac{\partial \chi(r, z)}{\partial r}= & \int_{0}^{\infty} \bar{\chi}(\xi, z) J_{0}(\xi r) \xi r d \xi \\
\frac{\partial^{2} \chi(r, z)}{\partial r^{2}}= & \int_{0}^{\infty} \bar{\chi}(\xi, z) J_{0}(\xi r) \xi d \xi \\
& +\int_{0}^{\infty}\left[-\bar{\chi}(\xi, z) \xi^{2}\right] J_{1}(\xi r) r d \xi
\end{aligned}
$$

$$
\frac{\partial^{2} \chi(r, z)}{\partial z^{2}}=\int_{0}^{\infty} \frac{\partial^{2} \bar{\chi}(\xi, z)}{\partial z^{2}} J_{1}(\xi r) r d \xi
$$


According to (15)-(17), the displacement can be expressed as follows:

$$
\begin{aligned}
v^{2} \chi & =\left(\frac{\partial}{\partial r^{2}}-\frac{1}{r} \frac{\partial}{\partial r}+\frac{\partial}{\partial z^{2}}\right) \chi(r, z) \\
& =\int_{0}^{\infty}\left[\frac{d^{2} \bar{\chi}(\xi, z)}{d z^{2}}-\xi^{2} \bar{\chi}(\xi, z)\right] J_{1}(\xi r) r d \xi \\
v^{2} v^{2} \chi & =\int_{0}^{\infty}\left[\frac{d^{2}}{d z^{2}}-\xi^{2}\right]^{2} \bar{\chi}(\xi, z) J_{1}(\xi r) r d \xi
\end{aligned}
$$

Combining (12) and (19) gives

$$
\int_{0}^{\infty}\left[\frac{d^{2}}{d z^{2}}-\xi^{2}\right]^{2} \bar{\chi}(\xi, z) J_{1}(\xi r) r d \xi=0
$$

Equation (20) can be rewritten as follows based on Hankel transform:

$$
\left[\frac{d^{2}}{d z^{2}}-\xi^{2}\right]^{2} \bar{\chi}(\xi, z)=0
$$

For a solution of the differential equation (21) yields the following general solution:

$$
\bar{\chi}(\xi, z)=\left(A_{\xi}+B_{\xi} z\right) e^{-\xi z}+\left(C_{\xi}+D_{\xi} z\right) e^{\xi z}
$$

Substituting (22) into (13) yields

$$
\begin{aligned}
& \chi(r, z)=\int_{0}^{\infty}\left[\left(A_{\xi}+B_{\xi} z\right) e^{-\xi z}+\left(C_{\xi}+D_{\xi} z\right) e^{\xi z}\right] \\
& \cdot J_{1}(\xi r) r d \xi .
\end{aligned}
$$

Substituting (22) into (17) and (18) yields

$$
\begin{gathered}
\frac{\partial^{2} \chi(r, z)}{\partial z^{2}}=\int_{0}^{\infty}\left\{\left[\xi^{2} A_{\xi}+\left(-2 \xi+\xi^{2} z\right) B_{\xi}\right] e^{-\xi z}\right. \\
\left.+\left[\xi^{2} C_{\xi}+\left(2 \xi+\xi^{2} z\right) D_{\xi}\right] e^{\xi z}\right\} J_{1}(\xi r) r d \xi \\
v^{2} \chi=\int_{0}^{\infty}\left(-2 \xi B_{\xi} e^{-\xi z}+2 \xi D_{\xi} e^{\xi z}\right) J_{1}(\xi r) r d \xi .
\end{gathered}
$$

Substituting (24)-(25) into (7)-(10), denoted by $A=$ $\xi^{2} A_{\xi}, B=\xi B_{\xi}, C=\xi^{2} C_{\xi}$, and $D=\xi D_{\xi}$, yields

$$
\begin{aligned}
\sigma_{r} & =\int_{0}^{\infty} \xi\left\{[A-(2-2 \mu-\xi z) B] e^{-\xi z}\right. \\
& \left.+[C+(2-2 \mu+\xi z) D] e^{\xi z}\right\} J_{0}(\xi r) d \xi-\frac{1}{r} U, \\
\sigma_{\theta} & =-2 \mu \int_{0}^{\infty} \xi\left(B e^{-\xi z}-D e^{\xi z}\right) J_{0}(\xi r) d \xi+\frac{1}{r} U, \\
\sigma_{z} & =-\int_{0}^{\infty} \xi\left\{[A+(2 \mu+\xi z) B] e^{-\xi z}\right. \\
& \left.+[C-(2 \mu-\xi z) D] e^{\xi z}\right\} J_{0}(\xi r) d \xi, \\
\tau_{z r} & =-\int_{0}^{\infty} \xi\left\{[A-(1-2 \mu-\xi z) B] e^{-\xi z}\right. \\
& \left.-[C+(1-2 \mu+\xi z) D] e^{\xi z}\right\} J_{1}(\xi r) d \xi, \\
u & =\frac{1+\mu}{E} U, \\
w & =\frac{1+\mu}{E} \int_{0}^{\infty}\left\{[A+(1+\xi z) B] e^{-\xi z}\right. \\
& \left.-[C-(1-\xi z) D] e^{\xi z}\right\} J_{0}(\xi r) d \xi,
\end{aligned}
$$

where $U=\int_{0}^{\infty}\left\{[A-(2-4 \mu-\xi z) B] e^{-\xi z}+[C+(2-4 \mu+\right.$ $\left.\xi z) D] e^{\xi z}\right\} J_{1}(\xi r) d \xi$

\section{The Relationship between General Solutions}

Parameters of Love solution are identified as $A_{L}, B_{L}, C_{L}$, and $D_{L}$, denoted by $A=A_{L}+(4 \mu-1) B_{L}, B=-B_{L}, C=C_{L}+(4 \mu-$ 1) $C_{L}$, and $D=D_{L}$; we will just get the general Love solution as follows:

$$
\begin{aligned}
\sigma_{r} & =-\int_{0}^{\infty} \xi\left\{\left[A_{L}-(1+2 \mu-\xi z) B_{L}\right] e^{-\xi z}\right. \\
& \left.-\left[C_{L}+(1+2 \mu+\xi z) D_{L}\right] e^{\xi z}\right\} J_{0}(\xi r) d \xi+\frac{1}{r} U_{L}, \\
\sigma_{\theta} & =2 \mu \int_{0}^{\infty} \xi\left(B_{L} e^{-\xi z}+D_{L} e^{\xi z}\right) J_{0}(\xi r) d \xi-\frac{1}{r} U_{L}, \\
\sigma_{z} & =\int_{0}^{\infty} \xi\left\{\left[A_{L}+(1-2 \mu+\xi z) B_{L}\right] e^{-\xi z}\right. \\
& \left.-\left[C_{L}-(1-2 \mu-\xi z) D_{L}\right] e^{\xi z}\right\} J_{0}(\xi r) d \xi, \\
\tau_{z r} & =\int_{0}^{\infty} \xi\left\{\left[A_{L}-(2 \mu-\xi z) B_{L}\right] e^{-\xi z}\right. \\
& \left.+\left[C_{L}+(2 \mu+\xi z) D_{L}\right] e^{\xi z}\right\} J_{1}(\xi r) d \xi, \\
u= & -\frac{1+\mu}{E} U_{L}, \\
w & +-\frac{1+\mu}{E} \int_{0}^{\infty}\left\{\left[C_{L}-(2-4 \mu-\xi z) D_{L}\right] e^{\xi z}\right\} J_{0}(\xi r) d \xi, \\
& \left.+(2-4 \mu+\xi z) B_{L}\right] e^{-\xi z}
\end{aligned}
$$


where $U_{L}=\int_{0}^{\infty}\left\{\left[A_{L}-(1-\xi z) B_{L}\right] e^{-\xi z}-\left[C_{L}+(1+\right.\right.$ $\left.\left.\xi z) D_{L}\right] e^{\xi z}\right\} J_{1}(\xi r) d \xi$.

Parameters of Southwell solution are identified as $A_{S}, B_{S}$, $C_{S}$, and $D_{S}$, denoted by $A=A_{S}+2 \mu B_{S}, B=-B_{S}, C=C_{S}+$ $2 \mu D_{S}$, and $D=D_{S}$; we will just get the general Southwell solution as follows:

$$
\begin{aligned}
\sigma_{r} & =\int_{0}^{\infty} \xi\left\{\left[A_{S}+(2-\xi z) B_{S}\right] e^{-\xi z}\right. \\
& \left.+\left[C_{S}+(2+\xi z) D_{S}\right] e^{\xi z}\right\} J_{0}(\xi r) d \xi-\frac{1}{r} U_{S}, \\
\sigma_{\theta} & =2 \mu \int_{0}^{\infty} \xi\left(B_{S} e^{-\xi z}+D_{S} e^{\xi z}\right) J_{0}(\xi r) d \xi+\frac{1}{r} U_{S}, \\
\sigma_{z} & =-\int_{0}^{\infty} \xi\left\{\left[A_{S}-\xi z B_{S}\right] e^{-\xi z}+\left[C_{S}+\xi z D_{S}\right] e^{\xi z}\right\} \\
& \cdot J_{0}(\xi r) d \xi, \\
\tau_{z r} & =-\int_{0}^{\infty} \xi\left\{\left[A_{S}+(1-\xi z) B_{S}\right] e^{-\xi z}\right. \\
& \left.-\left[C_{S}+(1+\xi z) D_{S}\right] e^{\xi z}\right\} J_{1}(\xi r) d \xi, \\
u & =\frac{1+\mu}{E} U_{S}, \\
w & =\frac{1+\mu}{E} \int_{0}^{\infty}\left\{\left[A_{S}-(1-2 \mu+\xi z) B_{S}\right] e^{-\xi z}\right. \\
& \left.-\left[C_{S}-(1-2 \mu-\xi z) D_{S}\right] e^{\xi z}\right\} J_{0}(\xi r) d \xi,
\end{aligned}
$$

where $U_{S}=\int_{0}^{\infty}\left\{\left[A_{S}+(2-2 \mu-\xi z) B_{S}\right] e^{-\xi z}+\left[C_{S}+(2-2 \mu+\right.\right.$ $\left.\left.\xi z) D_{S}\right] e^{\xi z}\right\} J_{1}(\xi r) d \xi$.

We can convert Southwell solution into Love solution, denoted by $A_{S}=-A_{L}-(2 \mu-1) B_{L}, B_{S}=B_{L}, C_{S}=C_{L}+$ $(2 \mu-1) D_{L}$, and $D_{S}=D_{L}$.

\section{The Present Solution Applies to Elastic Half-Space Foundation}

As shown in Figure 1, $p$ is vertical circular uniform distributed load; $\delta$ is radius of the circle. Displacement can be obtained under the load.

Boundary conditions are as follows:

$$
\begin{aligned}
z & =0, \\
\tau_{z r} & =0, \\
\sigma_{z} & = \begin{cases}-p & r \leq \delta \\
0 & r>\delta,\end{cases} \\
z & \longrightarrow \infty, \sigma_{z}, \\
u & =0 .
\end{aligned}
$$

Combining (25) and (30), we can get

$$
C=D=0 .
$$

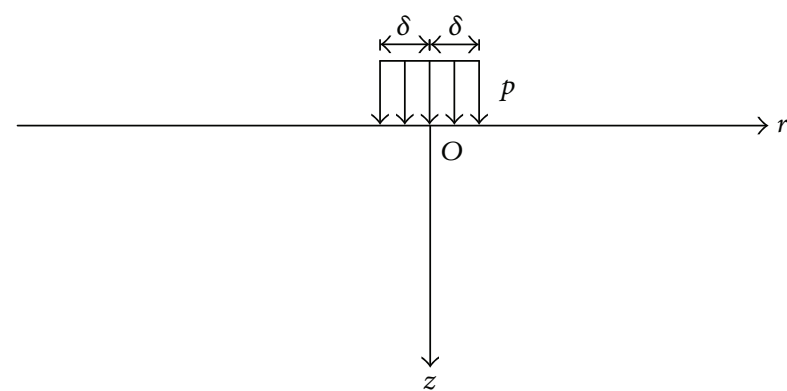

FIGURE 1: Load scheme.

From (29) and (30), denoted by $z=0$, we can get

$$
\begin{aligned}
& \sigma_{z}=-\int_{0}^{\infty} \xi(A+2 \mu B) J_{0}(\xi r) d \xi=-p \\
& \qquad(0 \leq r \leq \delta), \\
& \tau_{z r}=-\int_{0}^{\infty} \xi[A-(1-2 \mu) B] J_{1}(\xi r) d \xi=0 .
\end{aligned}
$$

Combining (29) and (32), we can get (33) based on Hankel transform:

$$
\begin{aligned}
& A=\frac{(1-2 \mu) p \delta J_{1}(\xi \delta)}{\xi}, \\
& B=\frac{p \delta J_{1}(\xi \delta)}{\xi} .
\end{aligned}
$$

Substituting (32) and (33) into (25) yields

$$
\begin{aligned}
w & =\frac{2 p a\left(1-\mu^{2}\right)}{E} \int_{0}^{\infty} \frac{J_{1}(x) J_{0}(x / \delta)}{x} d_{x} \\
& = \begin{cases}{ }_{2} F_{1}\left(\frac{1}{2},-\frac{1}{2} ; 1, \frac{r^{2}}{\delta^{2}}\right) & r<\delta \\
\frac{2}{\pi} & r=\delta \\
\frac{\delta}{2 r}{ }_{2} F_{1}\left(\frac{1}{2},-\frac{1}{2} ; 1, \frac{r^{2}}{\delta^{2}}\right) & r>\delta,\end{cases}
\end{aligned}
$$

where $x=\xi \delta$.

The result agreed with Love solution [1].

\section{The Present Solution Applies to Winkler Foundation}

5.1. Model. Circular uniform distributed load on Winkler foundation is as shown in Figure 2. 
TABLE 1: Displacement at point A.

\begin{tabular}{lcccccc}
\hline Integral interval & $0-10$ & $0-20$ & $0-30$ & $0-40$ & $0-50$ & $0-1000$ \\
\hline Displacement/mm & 0.844091 & 0.852095 & 0.85396 & 0.852095 & 0.852095 & 0.852479 \\
\hline
\end{tabular}

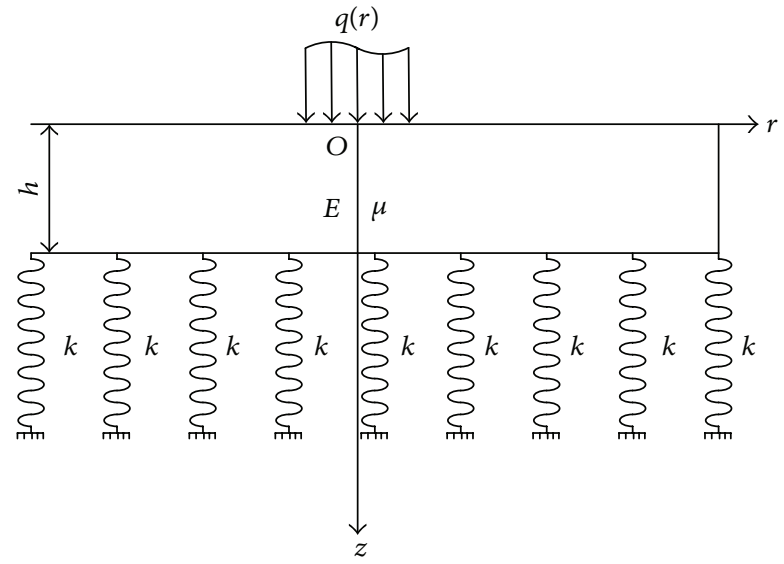

FIGURE 2: Calculation sketch map.

Boundary conditions are as follows:

$$
\begin{aligned}
z & =0, \\
\tau_{z r} & =0, \\
\sigma_{z} & = \begin{cases}-q(r) & r \leq \delta \\
0 & r>\delta,\end{cases} \\
z & =h, \\
\tau_{z r} & =0, \\
\sigma_{z} & =-k \omega(r) .
\end{aligned}
$$

Substituting (25) into (35) yields

$$
\begin{aligned}
\sigma_{z} & =-\int_{0}^{\infty} \xi(A+2 \mu B+C-2 \mu D) J_{0}(\xi r) d \xi \\
& =-q(r), \\
\tau_{z r} & =-\int_{0}^{\infty} \xi\{[A-(1-2 \mu) B]-[C+(1-2 \mu) D]\} \\
& \cdot J_{1}(\xi r) d \xi=0, \\
\sigma_{z} & =-\int_{0}^{\infty} \xi\left\{[A+(2 \mu+\xi h) B] e^{-\xi h}\right. \\
& \left.+[C-(2 \mu-\xi h) D] e^{\xi h}\right\} J_{0}(\xi r) d \xi=-k \omega(r), \\
\tau_{z r} & =-\int_{0}^{\infty} \xi\left\{[A-(1-2 \mu-\xi h) B] e^{-\xi h}\right. \\
& \left.-[C+(1-2 \mu+\xi h) D] e^{\xi h}\right\} J_{1}(\xi r) d \xi=0 .
\end{aligned}
$$

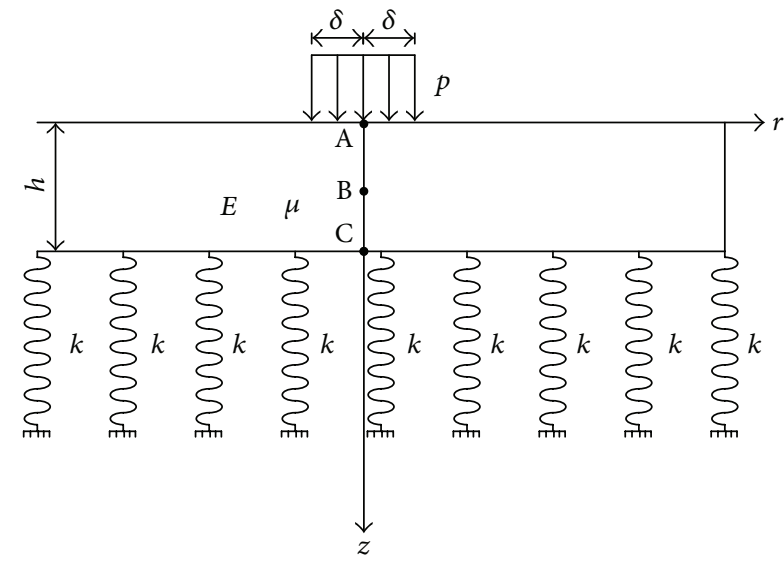

FIgURE 3: Calculation sketch map.

TABLE 2: Stress at point A.

\begin{tabular}{lcccccc}
\hline Integral interval & $0-10$ & $0-20$ & $0-30$ & $0-40$ & $0-50$ & $0-1000$ \\
\hline Stress/MPa & -1.39 & -1.83 & -1.86 & -1.74 & -1.69 & -1.69 \\
\hline
\end{tabular}

We can obtain (37) as follows based on Hankel transform:

$$
\begin{aligned}
& A+2 \mu B+C-2 \mu D=-\bar{q}(\xi), \\
& {[A-(1-2 \mu) B]-[C+(1-2 \mu) D]=0,} \\
& {[A+(2 \mu+\xi h) B] e^{-\xi h}} \\
& \quad+[C-(2 \mu-\xi h) D] e^{\xi h}=k \bar{\omega}(\xi), \\
& {[A-(1-2 \mu-\xi h) B] e^{-\xi h}} \\
& \quad-[C+(1-2 \mu+\xi h) D] e^{\xi h}=0,
\end{aligned}
$$

where $k$ is modulus of foundation reaction; $h$ is the thickness of the pavement.

The solution of (37) can obtain the expression for $A, B$, $C$, and $D$ about $\xi, \mu, E$, $h$, and $k$. Substituting $A, B, C$, and $D$ into (26), the stress and displacement of pavement can be obtained.

5.2. Examples. $h=0.2 \mathrm{~m}, E=10000 \mathrm{MPa}, \mu=0.15, k=1.5$ $\times 10^{7} \mathrm{~N} / \mathrm{m}^{3}, \delta=0.151 \mathrm{~m}$, and $q=700 \mathrm{KN} / \mathrm{m}^{2}$, as shown in Figure 3. We selected the integral intervals $0-10,0-20,0-$ $30,0-40,0-50$, and $0-1000$, respectively, and calculated displacement at point $\mathrm{A}$ and stress at points $\mathrm{A}, \mathrm{B}$, and $\mathrm{C}$; the results are summarized in Tables 1-4.

According to Tables 1, 2, 3, and 4, displacement at point $A$ is about $0.85 \mathrm{~mm}$, stress at point $\mathrm{A}$ is $-1.69 \mathrm{MPa}$, stress at point $\mathrm{B}$ is $-0.01 \mathrm{MPa}$, and stress at point $\mathrm{C}$ is $1.58 \mathrm{MPa}$. 
TABLE 3: Stress at point B.

\begin{tabular}{lcccccc}
\hline Integral interval & $0-10$ & $0-20$ & $0-30$ & $0-40$ & $0-50$ & $0-1000$ \\
\hline Stress/MPa & -0.008 & 0.007 & 0.009 & -0.002 & -0.001 & -0.001 \\
\hline
\end{tabular}

TABLE 4: Stress at point C.

\begin{tabular}{lcccccc}
\hline Integral interval & $0-10$ & $0-20$ & $0-30$ & $0-40$ & $0-50$ & $0-1000$ \\
\hline Stress $/ \mathrm{MPa}$ & 1.33 & 1.58 & 1.59 & 1.58 & 1.58 & 1.58 \\
\hline
\end{tabular}

\section{Conclusions}

A new form of the general solution of elastic space axisymmetric problem was obtained based on mathematics reasoning. The present solution can provide a new method for the elastic space axisymmetric contact problems, and Love solution and Southwell solution can be obtained by using variable substitution.

According to the boundary condition and characteristics, the present solution can be divided into solving boundary solution, stress solution, and displacement solution. Thus, the present solution would make a very nice complement to the elastic space axisymmetric contact problems.

\section{Competing Interests}

The authors declare that they have no competing interests.

\section{Acknowledgments}

This work was supported by the National Natural Science Foundation of China (Grant no. 51568044).

\section{References}

[1] Z. Chuanchao and W. Binggang, Mechanical Analysis of Road Structures, China Communications Press, Beijing, China, 2003.

[2] T. Kermanidis, "A numerical solution for axially symmetrical elasticity problems," International Journal of Solids and Structures, vol. 11, no. 4, pp. 493-500, 1975.

[3] D. S. Griffin and R. B. Kellogg, "A numerical solution for axially symmetrical and plane elasticity problems," International Journal of Solids and Structures, vol. 3, no. 5, pp. 781-794, 1967.

[4] A. E. Love, "The stress produced in a semi-infinite solid by pressure on part of the boundary," Philosophical Transactions of the Royal Society A, vol. 228, no. 659-669, pp. 377-420, 1929.

[5] V. S. Čemeris, "On the question of the numerical solution of the first basic problem of axisymmetric elasticity theory," Vychislitel'naya i Prikladnaya Matematika, no. 8, pp. 134-143, 1969.

[6] K. N. Bagisbaev, "Numerical solution of the AleksandrovSolov'ev integral equations for axially symmetric problems of elasticity theory," Izvestiya Akademii Nauk Kazakhskoui SSR. Seriya Fiziko-Matematicheskaya, no. 1, pp. 63-64, 1978.

[7] F. J. Rizzo and D. J. Shippy, "A boundary integral approach to potential and elasticity problems for axisymmetric bodies with arbitrary boundary conditions," Mechanics Research Communications, vol. 6, no. 2, pp. 99-103, 1979.
[8] R. D. Lazarov, "Numerical solution of some axisymmetric problems of the mathematical theory of elasticity by the finite difference method," Differentsial'nye Uravneniya, vol. 19, no. 3, pp. 500-507, 1983.

[9] A. N. Zlatin, "Exact solutions of the mixed axisymmetric problem of the torsion of an elastic space containing a spherical crack," PMM Journal of Applied Mathematics and Mechanics, vol. 76, no. 3, pp. 324-329, 2012.

[10] A. N. Zlatin, "The axisymmetric torsion problem for elastic space slackened by a spherical crack (Key dual series equations)," Doklady Physics, vol. 56, no. 5, pp. 268-270, 2011.

[11] E. Y. Mikhailova and G. V. Fedotenkov, "Nonstationary axisymmetric problem of the impact of a spherical shell on an elastic half-space (initial stage of interaction)," Mechanics of Solids, vol. 46, no. 2, pp. 239-247, 2011.

[12] V. I. Fabrikant, "Contact problems for several transversely isotropic elastic layers bonded to an elastic half-space," Journal of Applied Mathematics and Mechanics, vol. 91, no. 3, pp. 214246, 2011.

[13] D. G. Pavlou, "Elastodynamic analysis of a thin layer bonded on a visco-elastic medium under combined in-plane and lateral pulse loads," Mechanics Research Communications, vol. 38, no. 8, pp. 546-552, 2011.

[14] D. G. Pavlou, "Green's function for a pre-stressed thin plate on an elastic foundation under axisymmetric loading," Engineering Analysis with Boundary Elements, vol. 29, no. 5, pp. 428-434, 2005. 


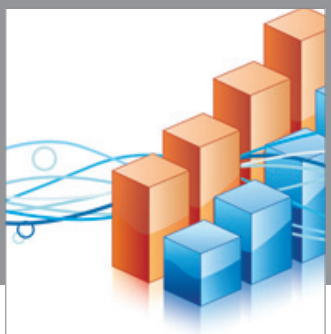

Advances in

Operations Research

vatem alat4

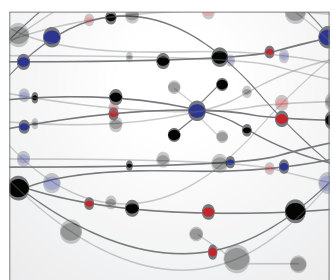

\section{The Scientific} World Journal
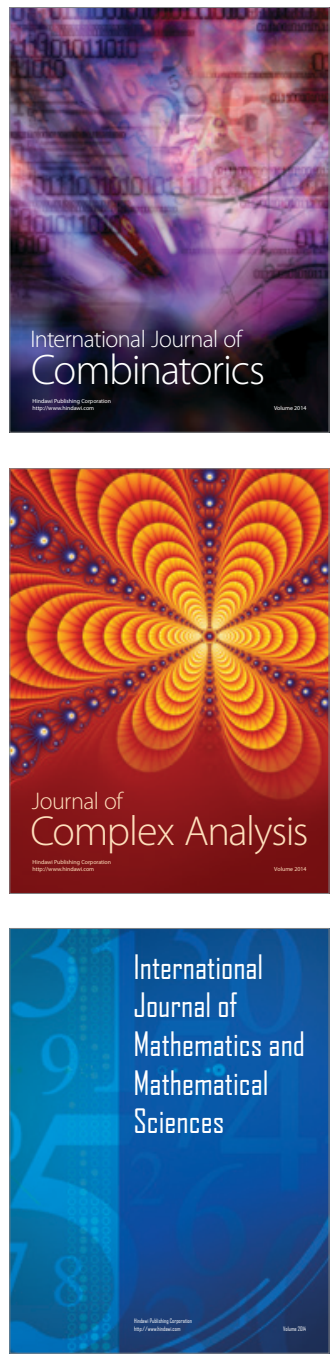
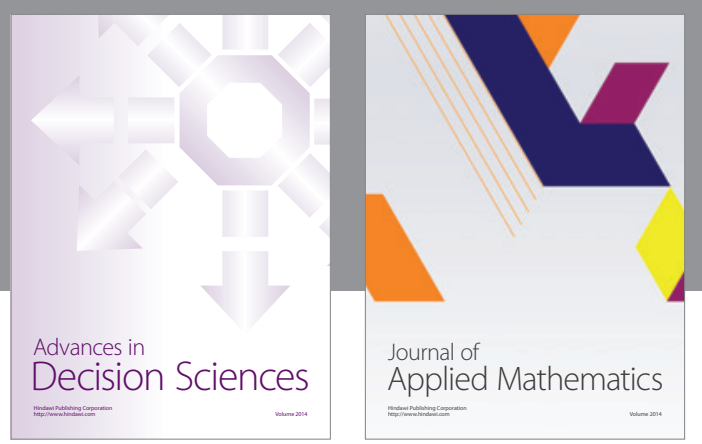

Algebra

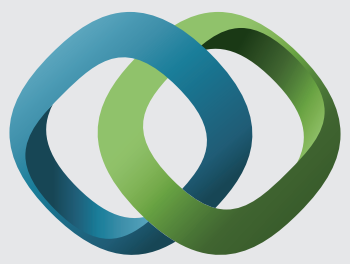

\section{Hindawi}

Submit your manuscripts at

http://www.hindawi.com
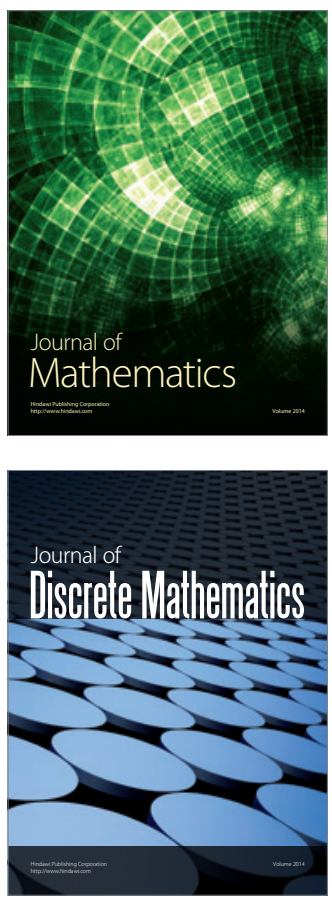

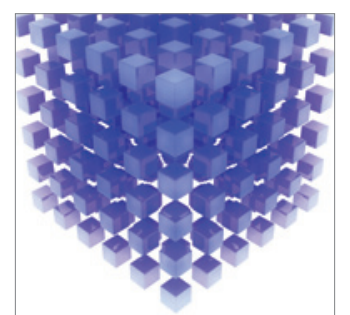

Mathematical Problems in Engineering
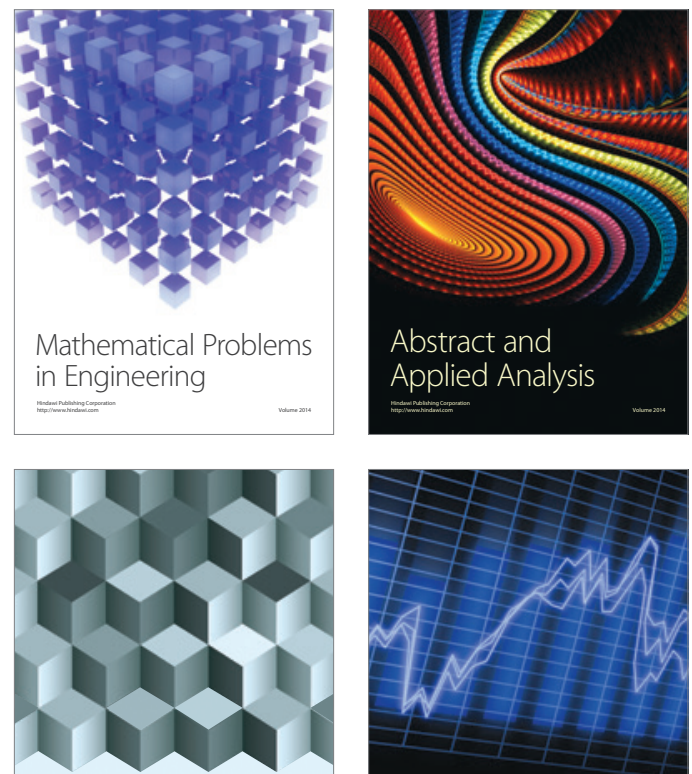

Journal of

Function Spaces

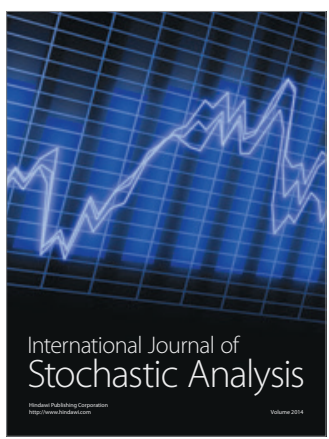

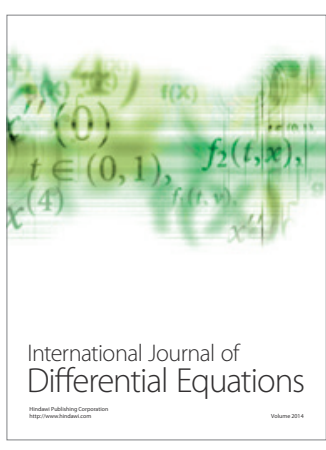
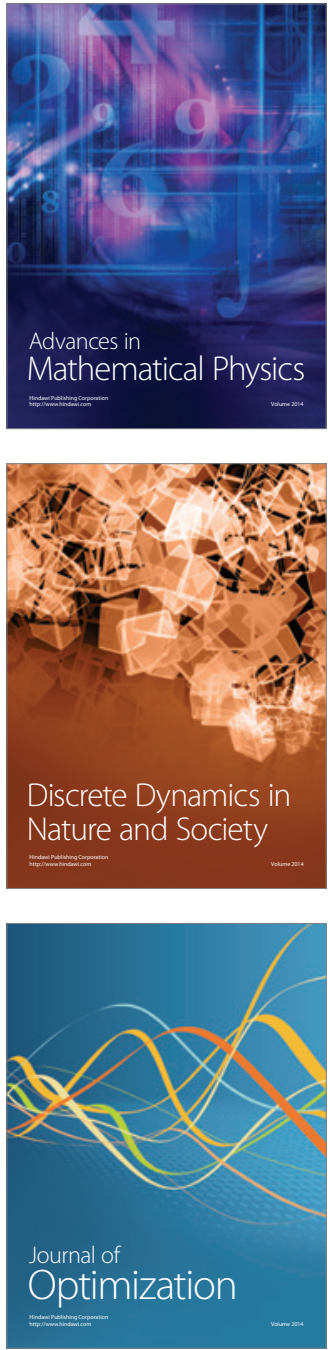